\title{
Lista de algas del Area de Protección Yum Balam, Quintana Roo, México
}

\author{
M.A. Díaz-Martín' ${ }^{1}$ E. Torres-Mejía ${ }^{2}$ y J. Espinoza-Avalos ${ }^{1}$ \\ ECOSUR, Unidad Chetumal. Apdo. Postal 424, Chetumal, Quintana Roo 77000, México. Fax 98320447, adiaz@ecosur- \\ qroo.mx \\ 2 SEMARNAP, km 4.8, Blvd. Kukulkán, Zona Hotelera, Cancún, Quintana Roo 77500, México.
}

Recibido 5-V-1997. Corregido 28-IV-1998. Aceptado 2-V-1998.

\begin{abstract}
A checklist on benthic marine algae of the Yum Balam Reserve, Quintana Roo, México, is presented. All species are "new records" for the area because this is the first study for the locality, 122 taxa were identified, 55 are species of Rhodophyta, 44 Chlorophyta and nine Phaeophyta. Batophora oerstedii v. occidentalis, Halodictyon mirabile, Hypoglossum involvens and Polysiphonia ramentacea are new records for the Yucatán Península.
\end{abstract}

Key words: Inventory, checklist, benthic algae, Yum Balam Area, Yucatán Península.

La flora ficológica del Caribe Mexicano es rica, a pesar de que aún existen áreas de ésta costa que no se han investigado florísticamente, incluyendo la Laguna Conil que en el año de 1994 es decretada como Area de Protección de la Flora y Fauna Silvestre y Acuática (APFFSA), nombrándola "Yum Balam". Entre los estudios más relevantes que se han llevado a cabo en el Caribe mexicano se encuentran los de Huerta (1958), Taylor $(1928,1972)$, de los cuales éste último se considera como la guía básica para cualquier trabajo de las algas del área, Huerta y Garza (1980), Mateo (1986), Huerta et al. (1987), Collado (1989), Mateo y Mendoza (1991), Mendoza y Mateo (1992), Collado (1992), Collado et al. (1995).

El presente trabajo es el primer aporte sobre la macroflora béntica de la Laguna Conil, resultado de un inventario de las macroalgas marinas rojas, verdes y pardas, lo que nos permitirá conocer mejor las áreas naturales sujetas a protección.

La Laguna Conil es peculiar porque se encuentra en la Península de Yucatán entre el Golfo de México y el Mar Caribe en una zona de transición oceanográfica en la parte norte del estado de Quintana Roo. La laguna es conocida también con el nombre de Yalahau o Ya'han y geográficamente forma parte del Golfo de México, a través de una barrera de arena denominada Isla Holbox. Esta laguna es muy importante por su gran diversidad faunística, incluyendo al "fósil viviente" Limulus polyphemus L. (Arthropoda), conocido como cacerolita de mar, especie endémica de las Penínsulas de Yucatán y Florida (Gómez 1993)

La zona de estudio es la continuación al Este de la Reserva Especial de la Biósfera de Ria Lagartos en el estado de Yucatán, siendo ésta una de las principales razones por la que fue 
decretada como Area de Protección de la Flora y Fauna Silvestre Acuática.

Las muestras se obtuvieron en abril de 1992 y septiembre de 1993, en temporada de secas y lluvias respectivamente; en cada temporada se realizaron recolectas generales en 11 puntos de la laguna, mediante buceo libre y SCUBA.

El material ficológico fue recolectado a mano o bien con la ayuda de una espátula para desprenderlo del sustrato duro, las plantas se colocaron en bolsas de plástico etiquetadas con los datos de recolecta y se fijaron en formol al $4 \%$ preparado con agua marina. Posteriormente se llevaron al laboratorio para su procesamiento e incorporación a la base de datos y colección ficológica del Herbario
CIQR de El Colegio de la Frontera Sur, unidad Chetumal.

La identificación del material ficológico se llevó a cabo utilizando las obras de Taylor (1928, 1972), Kim (1964), Joly (1967), y Woelkerling (1976). El arreglo taxonómico y actualización nomenclatural se realizó con base en el propuesto por Feldmann et al. (1975), Wynne (1986), Gabrielson y Cheney (1987), Aponte et al. (1994) y Silva et al. (1996).

Se identificó un total de 108 especies de algas marinas bénticas las cuales constituyen nuevos registros para el lugar ya que éste trabajo es el primero que se realiza para la zona de estudio; 44 especies, tres variedades y cinco formas corresponden a Chlorophyta, 55 especies a

\section{CUADRO 1}

Lista de especies encontradas en el Area de Protección de la Flora y Fauna Silvestre Yum Balam, Quintana Roo, indicando si fueron recolectadas en la temporada de secas y/o lluvias.

\section{Especie}

\section{RHODOPHYTA}

Florideophycidae'

Bonnemaisoniales

Bonnemaisoniaceae

Asparagopsis taxiformis (Delile) Trevisan

\section{Corallinales}

Corallinaceae

Amphiroa fragilissima (Linnaèus) Lamouroux

Jania adhaerens Lamouroux

J. pumila Lamouroux

J. rubens (Linnaeus) Lamouroux

Hypneaceae

H. musciformis (Wulfen in Jacquin) Lamouroux

H. spinella (C. Agardh) Kützing

H. valentiae (Turner) Montagne

Rhizophyllidaceae

Ochtodes secundiramea (Montagne) Howe

Caulacanthaceae

Catenella caespitosa (Withering) L. Irvine in Parke \& Dixon

C. impudica (Montagne) J. Agardh

Wurdemanniaceae

Wurdemannia miniata (Duby) J. Feldmann \& Hamel

Solieriacèae

Eucheuma isiforme (C. Agardh) J. Agardh

Meristiella gelidium (J. Agardh) Cheney \& Gabrielson

Solieria filiformis (Kützing) Gabrielson

Gracilariaceae

Gracilaria cervicornis (Turner) J. Agardh

$G$. verrucosa (Hudson) Papenfuss

Secas

Lluvias

$\mathrm{X}$

$\mathrm{X}$

$\mathrm{X}$

$\mathrm{X}$

$\mathrm{X}$

$\mathrm{X}$

$\mathrm{X}$

$\mathrm{X}$

$\mathrm{X}$

$\mathrm{X}$

$\mathrm{X}$

$\mathrm{X}$

$\mathrm{X}$
$\mathrm{X}$

$\mathrm{X}$

$\mathrm{X}$ 
Halymeniaceae

Grateloupia filicina (Lamouroux) C. Agardh

Rhodymeniales

Champiaceae

Champia parvula (C. Agardh) Harvey

C. salicornioides Harvey

Lomentaria baileyana (Harvey) Farlow

Rhodymeniaceae

Botryocladia occidentalis (Børgesen) Kylin

$B$. pyriformis (Børgesen) Kylin

Botryocladia sp. (J. Agardh) Kylin

Gelidiopsis intricata (C. Agardh) Vickers

Ceramiales

Ceramiaceae

Aglaothamnion cordatum (Børgesen) Feldmann- Mazoyer

$\mathrm{X}$

$\mathrm{X}$

$\mathrm{X}$

$\mathrm{X}$

$\mathrm{X}$

$\mathrm{X}$

$\mathrm{X}$

$\mathrm{X}$

$\mathrm{X}$

$\mathrm{X}$

$\mathrm{X}$

$\mathrm{X}$

$\mathrm{X}$

Ceramium cruciatum Collins \& Hervey

Crouania attenuata (C. Agardh) J. Agardh

Spyridia filamentosa (Wulfen) Harvey

Delesseriaceae

Caloglossa leprieurii (Montagne) G. Martens

Hypoglossum involvens (Harvey) J. A ardh

Dasyaceae

Dasya baillouviana (S.G. Gmelin) Montagne

D. caraibica Børgesen

D. rigidula (Kützing) Ardissone

Halodictyon mirabile Zanardini

Heterosiphonia crispella (C. Agardh) Wynne

H. gibbesii (Harvey) Falkenberg

Rhodomelaceae

Acanthophora muscoides (Linnaeus) Bory de Saint-Vincent

A. spicifera (Vahl) Børgesen

Bostrychia montagnei Harvey

B. tenella (Lamouroux) J. Agardh

Bryocladia cuspidata (J. Agardh) De Toni

Bryothamnion seaforthii (Turner) Kützing

Bryothamnion sp. Kützing

Chondria baileyana (Montagne) Harvey

C. capillaris (Hudson) Wynne

C. littoralis Harvey

Digenea simplex (Wulfen) C. Agardh

Laurencia intricata Lamouroux

L. microcladia Kützing

L. obtusa (Hudson) Lamouroux

L. papillosa (C. Agardh) Greville

L. poiteaui (Lamouroux) Howe

Murrayella periclados (C. Agardh) Schmitz

Polysiphonia ramentacea Harvey

Polysiphonia sp. Greville

\section{PHAEOPHYTA}

Dictyotales

Dictyotaceae

Dictyopteris justii Lamouroux 
D. divaricata Lamouroux

D. volubilis Kützing sensu Vickers

Padina boergesenii Allender \& Kraft

$P$. gymnospora (Kützing) Sonder

Padina sp. Adansson

Stypopodium zonale (Lamouroux) Papenfuss

Fucales

Sargassaceae

Sargassum sp. C. Agardh

\section{CHLOROPHYTA}

Siphonocladales

Siphonocladaceae

Cladophoropsis macromeres W. Taylor

C. membranacea (Hofman Bang ex C. Agardh) Børgesen Valoniaceae

Valonia macrophysa Kützing

V. utricularis (Roth) C. Agardh

Cladophorales

Anadyomenaceae

Anadyomene stellata (Wulfen) C. Agardh

Chaetomorpha gracilis Kützing

C. linum (O. F. Müller) Kützing

C. minima Collins \& Hervey

Rhizoclonium riparium (Roth) Harvey

Cladophoraceae

Cladophora sp. Kützing

Bryopsidales

Bryopsidaceae

Pedobesia lamourouxii (J. Agardh) J. Feldmann, Loreau, Codomier \& Couté

P. vaucheriaeformis (Harvey) J. Agardh

$$
\text { Codiaceae }
$$

Codium isthmocladum Vickers

Caulerpa ashmeadii Harvey

C. cupressoides (Vahl) C. Agardh

C. cupressoides $\mathrm{v}$. flabellata $\mathrm{B} \emptyset \mathrm{rgesen}$

C. mexicana Sonder ex Kützing

C. paspaloides (Bory de Saint-Vincent) Greville

C. prolifera (Forsskål) Lamouroux

C. prolifera f. obovata J. Agardh

C. racemosa (Forsskål) J. Agardh

C. racemosa v. peltata (Lamouroux) Eubank

C. sertularioides (S.G. Gmelin) Howe

C. sertularioides f. brevipes (J. Agardh) Svedelius

C. sertularioides $\mathrm{f}$. longiseta (Bory de Saint-Vincent) Svedelius

C. verticillata J. Agardh Udoteaceae

Avrainvillea longicaulis (Kützing) Murray \& Boodle

A. nigricans Decaisne

Boodleopsis pusilla (Collins) W. Taylor, Joly \& Bernatowicz

Penicillus capitatus Lamarck $x$
$x$
$x$ $x$ $\mathrm{X}$ $x$ $x$

$x$

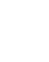


U. flabellum (Ellis \& Solander) Howe

$U$. spinulosa Howe

U. wilsoni A. Gepp, E. Gepp \& Howe Halimedaceae

Halimeda incrassata (Ellis) Lamouroux

H. monile (Ellis \& Solander) Lamouroux

H. opuntia (Linnaeus) Lamouroux

H. scabra Howe

H. tuna (Ellis \& Solander) Lamouroux

$$
\begin{aligned}
& \text { Dasycladales } \\
& \text { Dasycladaceae }
\end{aligned}
$$

Batophora oerstedii J. Agardh

B. oerstedii v. occidentalis (Harvey) Howe

Dasycladus vermicularis (Scopoli) Krasser

$\mathrm{X} \quad \mathrm{X}$

$\mathrm{X} \quad \mathrm{X}$

$\mathrm{X}-\mathrm{X}$

$\mathrm{X}-\mathrm{X}$

$\mathrm{X}$

$\mathrm{X}$

$\begin{array}{cc} & \mathrm{X} \\ & \mathrm{X} \\ \mathrm{X} & \mathrm{X} \\ & \mathrm{X} \\ & \mathrm{X} \\ & \\ & \\ & \mathrm{X} \\ & \mathrm{X} \\ \mathrm{X} & \mathrm{X} \\ \mathrm{X} & \mathrm{X} \\ & \mathrm{X} \\ & \mathrm{X}\end{array}$

Polyphysaceae

Acetabularia calyculus Lamouroux

A. crenulata Lamouroux

Polyphysa polyphysoides (P. \& H. Crouan in Mazé \& Schramm) Schnetter

\section{$\mathrm{X}$}

$\mathrm{X}$

$\mathrm{x}$

$x$

$x$

$\mathrm{X}$

Rhodophyta y nueve especies a Phaeophyta (Cuadro 1). Se encontró que las rodofitas se encuentran mejor representadas, con un $50.9 \%$ del total de las especies registradas, en comparación a las clorofitas con un $40.7 \%$ y las pardas con un $8.3 \%$. El bajo número de algas pardas presentes en este estudio concuerda con lo reportado para otras lagunas costeras de la costa del Pacífico Mexicano (Espinoza 1993). De las 108 especies de algas marinas identificadas en este estudio, tres especies y una variedad representan registros nuevos para la Península de Yucatán: Batophora oerstedii v. occidentalis, Halodictyon mirabile, Hypoglossum involvens y Polysiphonia ramentacea.

Para la temporada climática de lluvias, las macroalgas fueron más abundantes (100 taxa), en comparación a la temporada de secas (59), de manera similar a lo que anteriormente observaron Mateo y Mendoza (1991) y Mendoza y Mateo (1992).

Algunos de los ejemplares recolectados se dejaron a nivel de género ya que las características de las mismas no correspondieron al de las claves de identificación, o bien la muestra constaba de fragmentos del ejemplar lo que dificultó su identificación. Así mismo, cabe aclarar que debido a la escasés de estudios taxonómicos sobre la ficoflora de la región, es posible que algunas especies presenten problemas taxonómicos y nomenclaturales no detectados en el presente estudio.

\section{AGRADECIMIENTOS}

Los autores agradecen a Kurt M. Dreckmann-Estay por proporcionar la literatura pertinente para actualizar algunas de las nomenclaturas taxonómicas, así como a los revisores anónimos por sus valiosos comentarios y sugerencias al manuscrito.

\section{REFERENCIAS}

Aponte N.E., D.L. Ballantine \& J.N. Norris. 1994. Culture studies on the morphology and life history of Aglaothamnion herveyi (Howe) comb. nov., with notes on A. filipponei (Howe) comb. nov. (Ceramiaceae, Rhodophyta) Phycol. 33: 231-238.

Collado V.L. 1989. Estudio ecológico de las algas filamentosas como un grupo funcional de la Laguna de 
Bojórquez, Cancún, Quintana Roo, México. Tesis de Maestría. Instituto de Ciencias del Mar y Limnología. UNAM. México, D.F.

Collado V.L. 1992. Estudio fisionómico arquitectónico de las algas del sistema lagunar de Nichupté, Quintana Roo, México. Tesis de Doctorado. Facultad de Ciencias. UNAM. México, D.F.

Collado V.L; J. González G. \& E. Ezcurra. 1995. Patrones de distribución ficoflorística en el sistema lagunar de Nichupté, Quintana Roo, México. Act. Bot. Mex. 31: 19-32.

Espinoza A. J. 1993. Macroalgas Marinas del Golfo de California: p. 328-357. In (S.I. Salazar Vallejo y N.E. González, eds.). Biodiversidad Marina y Costera de México. Com. Nac. Biodiv.\& CIQRO. México.

Feldmann, J., J.P. Loreau, L. Codomier \& A. Couté. 1975. Morphologie et ultrastructure du esquelette des thalles calcifiés de Pedobesia (ex Derbesia) lamourouxii (J. Ag.) com. nov. C.R. Seances Acad. Sci. Ser. D. 280: 2641- 2644.

Gabrielson, P.G. \& D.P. Cheney. 1987. Morphology and taxonomy of Meristiella gen. nov. Solieriaceae, Rhodophyta) J. Phycol. 23: 481-493.

Gómez A.S. 1993. Cacerolita de Mar (Limulus polyphemus L.) en la Península de Yucatán. p. 650-659. In (S.1. Salazar Vallejo y N.E. González, eds.) Biodiversidad Marina y Costera de México. Com. Nac. Biodiv. \& CIQRO, México.

Huerta M.L. 1958. Contribución al conocimiento de los bajos de la sonda de Campeche, Cozumel e Isla Mujeres. An. Esc. Nac. Cienc. Biol. Méx. 10: 11-22.

Huerta M.L. \& M.A. Garza B. 1980. Contribución al conocimiento de la flora marina de la zona sur del litoral de Quintana Roo, México. An. Esc. Nac. Cienc. Biol. Méx. 23: 25-44.
Huerta M.L, A.C. Mendoza G. \& L.E. Mateo C. 1987. Avances sobre un estudio de las algas marinas de la Península de Yucatán. Phytol. 62 : 22-53.

Joly, A.B. 1967. Géneros da algas marinas da costa Atlántica latinoamericana. Universidad de Sao Paulo, Brasil. $461 \mathrm{p}$.

Kim, Ch.S. 1964. Marine Algae of Alacran Reef, Sourthern Gulf of Mexico. Tesis Doctoral. Universidad de Duke. United States of America.

Mateo C.L.E. 1986. Estudio florístico de las algas marinas bentónicas de Isla Cozumel, Quintana Roo, México. Tesis de Licenciatura. Instituto Politécnico Nacional. México, D.F.

Mateo C.L.E. \& A.C. Mendoza G. 1991. Algas marinas bénticas Cozumel, Quintana Roo, México. Act. Bot. Mex. 16:57-87.

Mendoza G.A.C. \& L.E. Mateo C. 1992. Algas marinas bentónicas de Isla Mujeres, Quintana Roo, México. Act. Bot. Mex. 19:37-61.

Silva, P.C., P. W. Basson \& R.L. Moe. 1996. Catalogue of the Benthic Marine Algae of the Indian Ocean. Univ. California, Berkeley. 1259 p.

Taylor, W.R. 1928. The marine algae of Florida with special reference to the Dry Tortugas. Institución Carnegie de Washington, D.C. 282 p.

Taylor, W.R. 1972. Marine Algae of the Eastern Tropical and Subtropical Coasts of the Ameicas. Universidad de Michigan, United States of America. 846 p.

Woelkerling, W.J. 1976. Keys and comments. Sedimenta. V. Division of marine geology and geophysics. Universidad de Miami. Miami, Florida. 476 p.

Wynne, M.J. 1986. A. Checklist of benthic marine algae of the tropical and subtropical Western Atlantic. Can. J. Bot. 64: 2239-2281. 